\title{
Resolving sets for higher dimensional projective spaces
}

\author{
Daniele Bartoli*, György Kissł Stefano Marcugini* and Fernanda Pambianco*
}

\begin{abstract}
Lower and upper bounds on the size of resolving sets for the point-hyperplane incidence graph of the finite projective space $\mathrm{PG}(n, q)$ are presented.
\end{abstract}

\section{Introduction}

For a connected, finite, simple graph $\Gamma=(V, E)$ and $x, y \in V$ let $d(x, y)$ denote the length of a shortest path joining $x$ and $y$.

Definition 1. Let $\Gamma=(V, E)$ be a finite, connected, simple graph. A vertex $v \in V$ is resolved by $S=\left\{v_{1}, \ldots, v_{n}\right\} \subset V$ if the ordered sequence $\left(d\left(v, v_{1}\right), d\left(v, v_{2}\right), \ldots, d\left(v, v_{n}\right)\right)$ is unique. $S$ is a resolving set in $\Gamma$ if it resolves all the elements of $V$. The metric dimension of $\Gamma$ is the size of the smallest example of resolving set in it.

The study of metric dimension is an interesting question in its own right and it is also motivated by the connection to the base size of the corresponding graph. The base size of a permutation group is the smallest number of points whose stabilizer is the identity. The base size of $\Gamma$ is the base size of its automorphism group. The study of base size dates back more than 50 years, see [10]. A resolving set for $\Gamma$ is obviously a base for $\operatorname{Aut}(\Gamma)$, so the metric dimension of a graph gives an upper bound on its base size.

The best known general bounds on metric dimension are given in the next theorem. The lower bound is straightforward, but the bound is tight (e.g. for a path or for a complete graph). The

${ }^{*}$ The research was supported by the Italian National Group for Algebraic and Geometric Structures and their Applications (GNSAGA - INDAM) and by University of Perugia, (Project: "Strutture Geometriche, Combinatoria e loro Applicazioni", Base Research Fund 2017).

${ }^{\dagger}$ The research was partially supported by the bilateral Slovenian-Hungarian Joint Research Project, grant no. NN 114614 (in Hungary) and N1-0032 (in Slovenia). 
upper bound was proved by Hernando et al. [7]. They also proved that for all integers $d \geq 2$ and $k \geq 1$, there exists a graph on $n$ vertices for which equality holds in Inequality (1).

Theorem 2 (Hernando et al.). If $\Gamma$ has $n$ vertices, its diameter is $d$ and its metric dimension is $k$, then

$$
k+d \leq n \leq\left(\left\lfloor\frac{2 d}{3}\right\rfloor+1\right)^{k}+k \sum_{i=1}^{\lceil d / 3\rceil}(2 i-1)^{k-1} .
$$

For more information about general results we refer the reader to the survey paper of Bailey and Cameron [1].

Much better bounds are known for incidence graphs of some linear spaces. In these cases, there are several estimates on the size of double blocking sets that can be used to prove lower bounds on the metric dimension. The knowledge of geometric properties are useful for the constructions and hence for the proof of upper bounds. It was shown by Héger and Takáts [6] that the metric dimension of the point-line incidence graph of a projective plane of order $q$ is $4 q-4$ if $q \geq 23$. Estimates on the metric dimension of affine and biaffine planes and generalized quadrangles were given by Bartoli et al. [2]. In higher dimensional spaces resolving sets are connected to lines in higgledy-piggledy arrangement which were studied by Fancsali and Sziklai [4].

Let $\Gamma_{P, \mathcal{H}}(n, q)$ be the point-hyperplane incidence graph of the finite projective space $\mathrm{PG}(n, q)$. The two sets of vertices of this bipartite graph correspond to the points and hyperplanes of PG $(n, q)$, respectively, and there is an edge between two vertices if and only if the corresponding point is in the corresponding hyperplane. In this paper, we investigate the metric dimension of $\Gamma_{P, \mathcal{H}}(n, q)$.

We use the same notations as in [6]. In particular

- $[P]$ and $[\mathcal{H}]$ denote the set of all hyperplanes through $P$ and all the points in the hyperplane $\mathcal{H}$, respectively;

- if $\mathcal{S}=\mathcal{P}_{S} \cup \mathcal{H}_{S}$, with $\mathcal{P}_{S}$ points and $\mathcal{H}_{S}$ hyperplanes, then inner points and inner hyperplanes indicate points or hyperplanes in $\mathcal{S}$, whereas outer points or outer hyperplanes denote points and hyperplanes not in $S$;

- an outer point is $t$-covered if it lies on exactly $t$ hyperplanes of $\mathcal{H}_{S}$.

In $\Gamma_{P, \mathcal{H}}(n, q)$ the distance of two distinct vertices is 2 if both vertices correspond to points or to hyperplanes and the distance is 1 or 3 if one vertex corresponds to a point and the other one corresponds to a hyperplane. Thus, outer points can only be resolved by hyperplanes and outer hyperplanes can only be resolved by points. Considering this property, the following definition is natural. 
Definition 3. A subset $S$ of vertices in $\Gamma_{P, \mathcal{H}}(n, q)$ is called a semi-resolving set for points (hyperplanes) if it resolves all of those vertices of $\Gamma_{P, \mathcal{H}}(n, q)$ that correspond to points (hyperplanes) of $\operatorname{PG}(n, q)$.

This paper is organized as follows. In Section 2, lower bounds on the size of resolving sets are proved in a pure combinatorial way. In Section 3, some constructions are presented. These resolving sets come from lines in higgledy-piggledy arrangement and the proof of our main result is based on estimates of the number of $\mathbb{F}_{q}$-rational points of suitable algebraic curves. Finally, in Section 4 computer aided result for spaces containing small number of points are given.

\section{A general lower bound}

Our first general lower bound is a generalization of the planar result of Héger and Takáts [6].

Theorem 4. If $q$ is large enough, then the size of any resolving set in $\Gamma_{P, \mathcal{H}}(n, q)$ is at least

$$
r=2 n q-2 \frac{n^{n-1}}{(n-1) !} .
$$

Proof. The statement for $n=2$ was proven by Héger and Takáts [6], so we may assume that $n>2$.

Suppose to the contrary that a resolving set $\mathcal{S}=\mathcal{P}_{S} \cup \mathcal{H}_{S}$ contains less elements than $r$. Because of the duality we may assume without loss of generality that $\mathcal{H}_{S}$ contains at most $(r-1) / 2$ hyperplanes. Let $\left|\mathcal{H}_{S}\right|=n q-C$, where $C>\frac{n^{n-1}}{(n-1) !}$. All outer points must be resolved by the elements of $\mathcal{H}_{S}$ because the distance of any two distinct points is exactly 2 . If $m<n$, then the intersection of any set of $m$ hyperplanes contains at least $q^{n-m}+q^{n-m-1}+\cdots+1>1$ points, thus any set of $m$ hyperplanes can resolve at most one $m$-covered points. Hence, a lot of points must be covered at least $n$ times. The maximum number of resolved $m$-covered points is $\left(\begin{array}{c}n q-C \\ m\end{array}\right)$, the number of 1-covered points and outer points altogether is $r$, and there is at most one not covered outer point. Therefore, counting the incident (inner hyperplane, point) pairs, we get

$$
\begin{aligned}
(n q-C)\left(q^{n-1}+q^{n-2}+\cdots+1\right) & \geq n\left(\frac{q^{n+1}-1}{q-1}-\sum_{m=2}^{n-1}\left(\begin{array}{c}
n q-C \\
m
\end{array}\right)-r-1\right)+\sum_{m=1}^{n-1} m\left(\begin{array}{c}
n q-C \\
m
\end{array}\right) \\
& =n\left(q^{n}+q^{n-1}+\cdots+1\right)-\sum_{i=1}^{n-2} i\left(\begin{array}{c}
n q-C \\
n-i
\end{array}\right)-n(r+1)+n q-C .
\end{aligned}
$$


The coefficients of $q^{n}$ are the same on the two sides of the inequality. As $n>2$ implies that the summands $n(r+1)$ and $n q$ do not contain any term of $q^{n-1}$, when comparing the coefficients of $q^{n-1}$ we get

$$
n-C \geq n-\frac{n^{n-1}}{(n-1) !}
$$

this contradiction proves the statement of the theorem.

Let us remark that in the case $n=2$ the bound is tight. Héger and Takáts [6] not only proved the lower bound if $q \geq 23$, but they also constructed several resolving sets of size $4 q-4$ for $\Gamma_{P, \mathcal{H}}(2, q)$, so we know that the metric dimension of the point-line incidence graph of a projective plane of order $q \geq 23$ is $4 q-4$.

In higher dimensions the sizes of the known resolving sets are much greater than the bound in Theorem 4. For instance, a construction due to Fancsali and Sziklai provides, under some assumptions on $n$ and $q$, resolving sets of size $(4 n-2) q$; see [4] and Theorem 10 below. From a result of Héger, Patkós and Takáts [5, Theorem 1.4], who used probabilistic methods, the existence of resolving sets of size $4(n+1) q$ follows. In the present paper, we construct resolving sets of sizes $8 q(n=3$ case $)$ and $12 q(n=4$ case), improving Theorem 10; see Theorem 9 and Corollary 12. Such constructions are still far from attaining the bound in Theorem 4 .

As outer hyperplanes can only be resolved by points, there is a natural connection between blocking sets and semi-resolving sets for hyperplanes and dually, between dual blocking sets and semi-resolving sets for points. The simplest $k$-fold blocking set for hyperplanes is the union of $k$ pairwise skew lines. If we restrict ourselves and we consider only those semi-resolving sets for hyperplanes that are contained in the union of some lines, then we can give much better estimates than the bound of Theorem 4. To do this, we recall a result of Fancsali and Sziklai [4, Lemma 13].

Lemma 5 (Fancsali and Sziklai, [4]). If the set $\mathcal{L}$ of lines in $\operatorname{PG}(n, q)$ has at most $\left\lfloor\frac{n}{2}\right\rfloor+n-1$ elements, then there exists a subspace of co-dimension 2 meeting each line in $\mathcal{L}$.

Corollary 6. If a semi-resolving set $S$ for hyperplanes of $\mathrm{PG}(n, q)$ is contained in the union of $m$ lines, then $m \geq\lfloor 3 n / 2\rfloor$.

Proof. Suppose to the contrary that $m<\lfloor 3 n / 2\rfloor$. By Lemma 5, there exists a subspace $\Pi$ of codimension 2 meeting each of the $m$ lines. This means that each of the $q+1$ hyperplanes containing $\Pi$ meets $S$ in the same set of points, contradiction. 


\section{Upper bounds}

In the planar case, all semi-resolving sets for lines are slight modifications of double blocking sets. The natural generalization of these constructions in $\operatorname{PG}(n, q)$ is the following: take an $n$-fold blocking set for hyperplanes and modify it a bit. We show that this idea does not work without requiring some additional properties. The simplest $n$-fold blocking set for hyperplanes is the point set contained in the union of $n$ pairwise skew lines. It could happen that a 2 -codimensional subspace, say $\Pi_{n-2}$, intersects each of the $n$ lines. In this case, any two hyperplanes whose intersection is $\Pi_{n-2}$ meet the $n$-fold blocking set in the same set of points, hence they are not resolved. To avoid this situation we need the notion of lines in higgledy-piggledy arrangement. This notion was introduced by Héger, Patkós and Takáts [5] and these sets were investigated by Fancsali and Sziklai [4].

Definition 7. In $\mathrm{PG}(n, q)$ a set of lines $\mathcal{L}$ is called a higgledy-piggledy arrangement if no 2codimensional subspace of $\mathrm{PG}(n, q)$ meets each element of $\mathcal{L}$.

The next lemma plays a crucial role later in our constructions.

Lemma 8. Suppose that $\mathrm{PG}(n, q)$ allows a set of $k$ lines in higgledy-piggledy arrangement. Then, the graph $\Gamma_{P, \mathcal{H}}(n, q)$ has a resolving set of size $2 k q$.

Proof. We construct a resolving set $\mathcal{S}=\mathcal{P}_{S} \cup \mathcal{H}_{S}$ having the extra property $\left|\mathcal{P}_{S}\right|=\left|\mathcal{H}_{S}\right|$. Because of duality, it is enough to show that there exists a set of points $\mathcal{P}_{S}$ of size $k q$ that resolves the hyperplanes of the space.

Let $\mathcal{L}=\left\{\ell_{1}, \ell_{2}, \ldots, \ell_{k}\right\}$ be a set of lines in higgledy-piggledy arrangement and $P_{i}$ be an arbitrary point on $\ell_{i}$. We claim that the set of points

$$
\mathcal{P}_{S}=\bigcup_{i=1}^{k}\left(\ell_{i} \backslash\left\{P_{i}\right\}\right)
$$

resolves the hyperplanes. Every hyperplane meets every line in either 1 or $q+1$ points. As there is no 2-codimensional subspace meeting each element of $\mathcal{L}$, and the intersection of any two hyperplanes is a 2 -codimensional subspace, we get that no two hyperplanes meet $\mathcal{P}_{S}$ in the same set of points, which proves the statement.

Our first result is based on a well-known property of hyperbolic quadrics in $\operatorname{PG}(3, q)$. This construction shows that the estimate in Corollary 6 is tight in the 3 -dimensional case.

Theorem 9. The graph $\Gamma_{P, \mathcal{H}}(3, q)$ has a resolving set of size $8 q$. 
Proof. By Lemma 8, it is enough to show that there exists a set of four lines which have no common transversal line.

Let $\mathcal{H}$ be a hyperbolic quadric, $\ell_{1}, \ell_{2}$ and $\ell_{3}$ be three pairwise skew lines on $\mathcal{H}$ and $\ell_{4}$ be an external line to $\mathcal{H}$. If a line contains three points of a quadratic surface, then each point of the line is on the surface. Thus, all the transversals of $\ell_{1}, \ell_{2}$ and $\ell_{3}$ are contained in $\mathcal{H}$. As the line $\ell_{4}$ has empty intersection with $\mathcal{H}$, there is no line meeting each of the four lines $\ell_{i}$. This proves the statement.

Fancsali and Sziklai [4] constructed sets of lines in higgledy-piggledy arrangement of size $2 n-1$ in $\mathrm{PG}(n, q)$ if $q$ satisfies some conditions. As a reformulation of [4, Theorem 20] we get the following statement.

Theorem 10 (Fancsali and Sziklai, [4]). If $q=p^{r}, p>n$ and $q \geq 2 n-1$, then the graph $\Gamma_{P, \mathcal{H}}(n, q)$ has a resolving set of size $(4 n-2) q$.

Our main result improves this bound for $n=4$. The proof is based on the following proposition which shows the existence of six lines in higgledy-piggledy arrangement in PG(4,q). Let us remark that our construction is totally different from the one of Fancsali and Sziklai, although we also use the Grassmann coordinates. For a brief introduction to these coordinates we refer the reader to [9, Section 24.1].

Proposition 11. In $\mathrm{PG}\left(4, p^{r}\right)$ if $p \neq 2, p \neq 3$ and $q>36086$, then there exists $\alpha \in \mathrm{GF}\left(p^{r}\right)$ such that there is no plane which intersects each of the six lines joining the pairs of points

$$
\begin{array}{lll}
(1: 0: 0: 0: 0) & \text { and } & (0: 1: 1: 0: 0) \\
(0: 1: 0: 0: 0) & \text { and } & (0: 0: 1: 1: 0) \\
(0: 0: 1: 0: 0) & \text { and } & (0: 0: 0: 1: 1) \\
(0: 0: 0: 1: 0) & \text { and } & (1: 0: 0: 0: 1) \\
(0: 0: 0: 0: 1) & \text { and } & (1: 1: 0: 0: 0) \\
(1: 1: 1: 1: 1) & \text { and } & (1: 0: 1: 0: 0)
\end{array}
$$

Proof. The Grassmann coordinates

$$
\left(g_{01}: g_{02}: g_{03}: g_{04}: g_{12}: g_{13}: g_{14}: g_{23}: g_{24}: g_{34}\right)
$$


of the lines are the following.

$$
\begin{array}{ll}
\ell_{1}: & (1: 1: 0: 0: 0: 0: 0: 0: 0: 0), \\
\ell_{2}: & (0: 0: 0: 0: 1: 1: 0: 0: 0: 0), \\
\ell_{3}: & (0: 0: 0: 0: 0: 0: 0: 1: 1: 0), \\
\ell_{4}: & (0: 0: 1: 0: 0: 0: 0: 0: 0:-1), \\
\ell_{5}: & (0: 0: 0: 1: 0: 0: 1: 0: 0: 0), \\
\ell_{6}: & (1: 0: 1-\alpha: 1:-1:-\alpha: 0: 1-\alpha: 1: \alpha) .
\end{array}
$$

Suppose that a plane $\Pi$ with Grassmann coordinates

$$
\left(g_{234}: g_{314}: g_{124}: g_{213}: g_{034}: g_{204}: g_{023}: g_{014}: g_{103}: g_{012}\right)
$$

meets each of the six lines. This means that the dot product of the coordinate vectors of $\ell_{i}$ and $\Pi$ is zero. Writing it for $i=1,2, \ldots 5$ we get $g_{234}+g_{314}=0, g_{034}+g_{204}=0, g_{014}+g_{103}=0$, $g_{124}-g_{012}=0$ and $g_{213}+g_{023}=0$, respectively. Let $g_{234}=a, g_{123}=b, g_{213}=c, g_{034}=d g_{014}=e$. Then, the coordinate vector of $\Pi$ is

$$
(a:-a: b: c: d:-d:-c: e:-e: b) .
$$

This vector satisfies the quadratic Plücker relations

$$
g_{i_{1} i_{2} i_{3}} g_{j_{1} j_{2} j_{3}}=g_{j_{1} i_{2} i_{3}} g_{i_{1} j_{2} j_{3}}-g_{j_{2} i_{2} i_{3}} g_{i_{1} j_{1} j_{3}},
$$

thus choosing $j_{3}=4,3,2,1$ and 0 we get

$$
a e=a d-b d, \quad a c=c d-a e, \quad a b=c d-b c, \quad a b=b e+c e, \quad b d=c e+d e,
$$

respectively. The plane $\Pi$ also meets $\ell_{6}$, hence we have $0=a+(1-\alpha) b+c-d+\alpha d+(1-\alpha) e-e+\alpha b$, so

$$
a+b+c+(\alpha-1) d-\alpha e=0
$$

We claim that for a suitable choice of $\alpha$ Equations (2) and (3) imply $a=b=c=d=e=0$.

First, suppose that $a=0$. Then, from the first three equations of (2) we get $b d=c d=b c=0$, hence at least two of $b, c$ and $d$ are 0 . Then, the fifth equation of (2) implies that either $b=c=d=0$, or $e=0$, so there is at most one non-zero among $a, b, c, d$ and $e$. This fact, together with Equation (3), prove the statement of the theorem.

Now, suppose that $a \neq 0$. Because of the homogeneity we may assume without loss of generality that $a=1$. 
If $d=0$, then the first and second equations of (2) imply $e=0$ and $c=0$, and the third equation implies $b=0$, because $a=1$. Thus, Equation (3) gives $1=0$, contradiction.

If $d=1$, then the second equation of (2) gives $e=0$, hence the first equation of (2) implies $b=1$. This means that the third equation of (2) becomes $1=0$, a contradiction again.

Suppose that $0 \neq d \neq 1$. Then, the first and second equations of (2) give $b=\frac{d-e}{d}$ and $c=\frac{e}{d-1}$, respectively. Substituting these expressions into the remaining three equations of (2), we get

$$
d^{2} e-d^{2}+d+e^{2}-e=0
$$

in each case, while substituting into Equation (3) gives

$$
(1-\alpha) d^{3}+(\alpha-3) d^{2}+2 d+e\left(\alpha d^{2}-\alpha d-1\right)=0 .
$$

The resultant of these two equations with respect to $e$ is

$$
\left(d^{5}-d^{4}\right) \alpha^{2}+\left(-d^{5}+d^{4}+4 d^{3}-5 d^{2}+d\right) \alpha+d^{4}-4 d^{3}+6 d^{2}-5 d+1 .
$$

In the following, we prove that there exists at least one $\alpha \in \mathbb{F}_{q}$ such that Equation (4) has no solution in $\mathbb{F}_{q}$.

First of all, we count the number of $\alpha$ s for which the equation

$$
G_{\alpha}(d)=\left(d^{5}-d^{4}\right) \alpha^{2}+\left(-d^{5}+d^{4}+4 d^{3}-5 d^{2}+d\right) \alpha+d^{4}-4 d^{3}+6 d^{2}-5 d+1=0
$$

has at least two distinct solutions in $\mathbb{F}_{q}$. Consider the polynomial

$$
F_{x, y, z, t, u}(d)=(d+x)(d+y)\left(d^{3}+d^{2} z+d t+u\right) \in \mathbb{F}_{q}[d] .
$$

We will give a lower bound on the number of six-tuples $[x, y, z, t, u, \alpha] \in \mathbb{F}_{q}^{6}$ such that $G_{\alpha}(d)=$ $\lambda F_{x, y, z, t, u}(d)$ for some $\lambda \in \mathbb{F}_{q}^{*}$.

This immediately gives $\lambda=\alpha^{2}-\alpha$, therefore $\alpha \neq 0,1$, and

$$
\left\{\begin{array}{l}
x y u \alpha^{2}-x y u \alpha-1=0 \\
x \alpha^{2}-x \alpha+y \alpha^{2}-y \alpha+z \alpha^{2}-z \alpha+\alpha^{2}-\alpha-1=0 \\
x y t \alpha^{2}-x y t \alpha+x u \alpha^{2}-x u \alpha+y u \alpha^{2}-y u \alpha-\alpha+5=0 \\
x y z \alpha^{2}-x y z \alpha+x t \alpha^{2}-x t \alpha+y t \alpha^{2}-y t \alpha+u \alpha^{2}-u \alpha+5 \alpha-6=0 \\
x y \alpha+x z \alpha+y z \alpha+t \alpha-4=0 .
\end{array} .\right.
$$

We also require that $x \neq y$, that is $x-y \neq 0$, and $x y \neq 0$ in order to consider the following substitution:

$$
\begin{aligned}
& t=-(x y \alpha+x z \alpha+y z \alpha-4) / \alpha, \\
& u=1 /\left(x y\left(\alpha^{2}-\alpha\right)\right), \\
& z=-\left(x \alpha^{2}-x \alpha+y \alpha^{2}-y \alpha+\alpha^{2}-\alpha-1\right) /\left(\alpha^{2}-\alpha\right) .
\end{aligned} .
$$


We get

$$
\left\{\begin{aligned}
0= & \left(x^{4} y^{2}+x^{3} y^{3}+x^{3} y^{2}+x^{2} y^{4}+x^{2} y^{3}\right) \alpha^{2} \\
& +\left(-x^{4} y^{2}-x^{3} y^{3}-x^{3} y^{2}-x^{2} y^{4}-x^{2} y^{3}+4 x^{2} y^{2}-x y\right) \alpha \\
& -x^{3} y^{2}-x^{2} y^{3}-4 x^{2} y^{2}+5 x y+x+y \\
0= & \left(x^{4} y+x^{3} y^{2}+x^{3} y+x^{2} y^{3}+x^{2} y^{2}+x y^{4}+x y^{3}\right) \alpha^{2} \\
& +\left(-x^{4} y-x^{3} y^{2}-x^{3} y-x^{2} y^{3}-x^{2} y^{2}+4 x^{2} y-x y^{4}-x y^{3}+4 x y^{2}+5 x y\right) \alpha \\
& -x^{3} y-x^{2} y^{2}-4 x^{2} y-x y^{3}-4 x y^{2}-6 x y+1 .
\end{aligned}\right.
$$

It is easily seen that $\alpha \neq 0,1$ is equivalent to

$$
\left(x^{3} y^{2}+x^{2} y^{3}-4 x y-x-y\right)\left(x^{3} y^{2}+x^{2} y^{3}+4 x^{2} y^{2}-5 x y-x-y\right) \neq 0 .
$$

Now, the resultant of the two polynomials in (5) with respect to $\alpha$ contains the factor $H(x, y)$ equal to

$$
\begin{aligned}
& x^{8} y^{8}+4 x^{8} y^{7}+7 x^{8} y^{6}+13 x^{8} y^{5}+24 x^{8} y^{4}+33 x^{8} y^{3}+27 x^{8} y^{2}+9 x^{8} y+x^{8}+4 x^{7} y^{8}+11 x^{7} y^{7}+8 x^{7} y^{6} \\
& +23 x^{7} y^{5}+79 x^{7} y^{4}+110 x^{7} y^{3}+71 x^{7} y^{2}+20 x^{7} y+2 x^{7}+7 x^{6} y^{8}+8 x^{6} y^{7}-3 x^{6} y^{6}+87 x^{6} y^{5}+262 x^{6} y^{4} \\
& +256 x^{6} y^{3}+101 x^{6} y^{2}+17 x^{6} y+x^{6}+13 x^{5} y^{8}+23 x^{5} y^{7}+87 x^{5} y^{6}+367 x^{5} y^{5}+586 x^{5} y^{4}+397 x^{5} y^{3} \\
& +112 x^{5} y^{2}+11 x^{5} y+24 x^{4} y^{8}+79 x^{4} y^{7}+262 x^{4} y^{6}+586 x^{4} y^{5}+622 x^{4} y^{4}+307 x^{4} y^{3}+70 x^{4} y^{2}+6 x^{4} y \\
& +33 x^{3} y^{8}+110 x^{3} y^{7}+256 x^{3} y^{6}+397 x^{3} y^{5}+307 x^{3} y^{4}+104 x^{3} y^{3}+16 x^{3} y^{2}+x^{3} y+27 x^{2} y^{8}+71 x^{2} y^{7} \\
& +101 x^{2} y^{6}+112 x^{2} y^{5}+70 x^{2} y^{4}+16 x^{2} y^{3}+x^{2} y^{2}+9 x y^{8}+20 x y^{7}+17 x y^{6}+11 x y^{5}+6 x y^{4}+x y^{3}+y^{8}+2 y^{7}+y^{6} .
\end{aligned}
$$

Let $\beta=\alpha+\frac{x^{3} y+x^{2} y^{2}+x^{2} y+x y^{3}+x y^{2}-4 x y+1}{2 x y\left(x^{2}+x y+x+y^{2}+y\right)}$. Then

$$
\begin{aligned}
4 x^{2} y^{2}\left(x^{2}+x y+x+y^{2}+y\right)^{2} \beta^{2}= & x^{6} y^{2}+2 x^{5} y^{3}+6 x^{5} y^{2}+3 x^{4} y^{4} \\
& +12 x^{4} y^{3}+13 x^{4} y^{2}+2 x^{3} y^{5}+12 x^{3} y^{4}+18 x^{3} y^{3}+8 x^{3} y^{2} \\
& -18 x^{3} y-4 x^{3} x^{2} y^{6}+6 x^{2} y^{5}+13 x^{2} y^{4}+8 x^{2} y^{3}-2 x^{2} y^{2} \\
& -26 x^{2} y-4 x^{2}-18 x y^{3}-26 x y^{2}-16 x y-4 y^{3}-4 y^{2}+1 .
\end{aligned}
$$


Finally, put $\gamma=2 x y\left(x^{2}+x y+x+y^{2}+y\right) \beta$. Then, we obtain

$$
\begin{aligned}
\gamma^{2}= & x^{6} y^{2}+2 x^{5} y^{3}+6 x^{5} y^{2}+3 x^{4} y^{4}+12 x^{4} y^{3}+13 x^{4} y^{2}+2 x^{3} y^{5}+12 x^{3} y^{4}+18 x^{3} y^{3} \\
& +8 x^{3} y^{2}-18 x^{3} y-4 x^{3}+x^{2} y^{6}+6 x^{2} y^{5}+13 x^{2} y^{4}+8 x^{2} y^{3}-2 x^{2} y^{2}-26 x^{2} y \\
& -4 x^{2}-18 x y^{3}-26 x y^{2}-16 x y-4 y^{3}-4 y^{2}+1 \\
= & L(x, y) .
\end{aligned}
$$

Now, consider the algebraic variety $\mathcal{V}$ defined by Equation $(6)$ and $H(x, y)=0$. We are going to prove the existence of an absolutely irreducible component defined over $\mathbb{F}_{q}$ of $\mathcal{V}$. Consider the birational transformation $\varphi$ defined as $\varphi(x, y, \gamma)=\left(x, x^{2} y-1, \gamma\right)$. Let $H^{\prime}(x, y)=H(\varphi(x, y, \gamma))$ and $\gamma^{2}=L(\varphi(x, y, \gamma))$. It is easily seen that the new variety $\mathcal{V}^{\prime}$ has a component given by $\gamma^{2}=$ $L(\varphi(x, y, \gamma)), H(\varphi(x, y, \gamma)) / x^{4}=0$. This component contains a simple $\mathbb{F}_{q \text {-rational point }(0: 0: 1)}$ and therefore there exists an absolutely irreducible $\mathbb{F}_{q}$-rational component $\mathcal{X}$ through it which is not contained in the plane $x=0$. The variety $\mathcal{V}^{\prime}$ has equations

$$
\left\{\begin{aligned}
0= & x^{20} y^{8}+4 x^{19} y^{8}+7 x^{18} y^{8}-4 x^{18} y^{7}+13 x^{17} y^{8}-21 x^{17} y^{7}+24 x^{16} y^{8}-48 x^{16} y^{7}+7 x^{16} y^{6}+33 x^{15} y^{8} \\
& -81 x^{15} y^{7}+43 x^{15} y^{6}+27 x^{14} y^{8}-113 x^{14} y^{7}+137 x^{14} y^{6}-x^{14} y^{5}+9 x^{13} y^{8}-154 x^{13} y^{7}+290 x^{13} y^{6} \\
& -18 x^{13} y^{5}+x^{12} y^{8}-145 x^{12} y^{7}+381 x^{12} y^{6}-119 x^{12} y^{5}-6 x^{12} y^{4}-52 x^{11} y^{7}+410 x^{11} y^{6}-400 x^{11} y^{5} \\
& -21 x^{11} y^{4}-6 x^{10} y^{7}+360 x^{10} y^{6}-671 x^{10} y^{5}-8 x^{10} y^{4}+11 x^{10} y^{3}+129 x^{9} y^{6}-677 x^{9} y^{5}+161 x^{9} y^{4} \\
& +25 x^{9} y^{3}+15 x^{8} y^{6}-515 x^{8} y^{5}+537 x^{8} y^{4}+26 x^{8} y^{3}-9 x^{8} y^{2}-175 x^{7} y^{5}+622 x^{7} y^{4}+60 x^{7} y^{3} \\
& -14 x^{7} y^{2}-20 x^{6} y^{5}+430 x^{6} y^{4}-140 x^{6} y^{3}+18 x^{6} y^{2}+x^{6} y+136 x^{5} y^{4}-272 x^{5} y^{3}-47 x^{5} y^{2}+4 x^{5} y \\
& +15 x^{4} y^{4}-191 x^{4} y^{3}-36 x^{4} y^{2}-12 x^{4} y+x^{4}-57 x^{3} y^{3}+30 x^{3} y^{2}+4 x^{3} y-6 x^{2} y^{3}+33 x^{2} y^{2}+18 x^{2} y \\
& +10 x y^{2}+8 x y+y^{2}+y \\
\gamma^{2}= & x^{14} y^{6}+2 x^{13} y^{5}+3 x^{12} y^{4}+2 x^{11} y^{4}+2 x^{11} y^{3}-2 x^{10} y^{4}+x^{10} y^{2}-10 x^{9} y^{3}-4 x^{8} y^{3}-5 x^{8} y^{2}-2 x^{8} y \\
& -18 x^{7} y^{3}+6 x^{7} y^{2}-6 x^{7} y-4 x^{6} y^{3}+7 x^{6} y^{2}-2 x^{6} y+x^{6}+28 x^{5} y^{2}-18 x^{5} y+4 x^{5}+8 x^{4} y^{2}-26 x^{4} y \\
& +4 x^{4}-18 x^{3} y+14 x^{3}-4 x^{2} y+20 x^{2}+8 x+1 .
\end{aligned}\right.
$$

Let $\mathcal{Y}=\varphi^{-1}(\mathcal{X})$ be the corresponding $\mathbb{F}_{q}$-rational component in $\mathcal{V}$. In order to estimate the genus of $\mathcal{Y}$, we notice that the curve defined by $H(x, y)=0$ has genus at most $105-56=49$, since such a curve has degree 16 and at least two ordinary singular points of multiplicity 8 (the two ideal points). Thus, by [11, Corollary 3.7.4],

$$
g(\mathcal{Y}) \leq 1+2(49-1)+\frac{1}{2}(128)=161
$$

Hence, from the Hasse-Weil Bound [11, Theorem 5.2.3], we get that $\mathcal{Y}$ has at least

$$
q-161 \sqrt{q}-3
$$


affine $\mathbb{F}_{q}$-rational points.

Recall that we need to prove the existence of $\mathbb{F}_{q}$-rational points $\left(x_{0}: y_{0}: \gamma_{0}\right)$ such that $g\left(x_{0}, y_{0}\right) \neq 0$, where

$g\left(x_{0}, y_{0}\right)=x_{0} y_{0}\left(x_{0}-y_{0}\right)\left(x_{0}^{3} y_{0}^{2}+x_{0}^{2} y_{0}^{3}-4 x_{0} y_{0}-x_{0}-y_{0}\right)\left(x_{0}^{3} y_{0}^{2}+x_{0}^{2} y_{0}^{3}+4 x_{0}^{2} y_{0}^{2}-5 x_{0} y_{0}-x_{0}-y_{0}\right)$,

since the corresponding $\alpha$ must be different from 0 and 1 . Now, the variety given by $g(x, y)=0$ is mapped by $\varphi(x, y, \gamma)$ into a variety of degree $30 \mathrm{in} x, y$. The component $\mathcal{V}^{\prime}$ can have at most $30 \cdot 28 \cdot 2=1680$ points in common with $g \circ \varphi$. Summing up, there are at least $q-161 \sqrt{q}-1683$ suitable triples $\left(x_{0}, y_{0}, \gamma_{0}\right)$, which correspond to suitable 6-tuples $\left(x_{0}, y_{0}, z_{0}, t_{0}, u_{0}, \alpha_{0}\right)$ such that $G_{\alpha}(d)$ has at least 2 distinct roots in $\mathbb{F}_{q}$. Since each polynomial $G_{\alpha}(d)$ can correspond to at most to $5 \cdot 4=206$-tuples, the argument above proves the existence of at least $(q-161 \sqrt{q}-1683) / 20$ values of $\alpha$ such that the corresponding $G_{\alpha}(d)$ has at least 2 distinct roots in $\mathbb{F}_{q}$.

Now, each pair $(d, \alpha) \in \mathbb{F}_{q}^{2}$ such that $G_{\alpha}(d)=0$ corresponds to an $\mathbb{F}_{q}$-rational point of the curve $\mathcal{Z}$ defined by $G_{\alpha}(d)=0$. Since such a curve has genus at most 1 , there exist at most $q+2 \sqrt{q}-1$ such pairs, again by [11, Theorem 5.2.3]. Finally, the number of $\bar{\alpha} \in \mathbb{F}_{q} \backslash\{0,1\}$ such that $G_{\bar{\alpha}}(d)$ has no roots, or equivalently no points $(d, \bar{\alpha})$ belong to the curve $G_{\alpha}(d)=0$, is lower bounded by

$$
\underbrace{q-2}_{\left|\mathbb{F}_{q} \backslash\{0,1\}\right|}-(\underbrace{q+2 \sqrt{q}-1}_{|\mathcal{Z}|}-2 \underbrace{\frac{q-161 \sqrt{q}-1683}{20}}_{\text {"good" 6-tuples }})=\frac{q-181 \sqrt{q}-1683}{10} .
$$

If $q>36086$, then the previous quantity is larger than 1 and the existence of a suitable $\alpha$ is proved.

Corollary 12. The graph $\Gamma_{P, \mathcal{H}}(4, q)$ has a resolving set of size $12 q$.

Proof. By Lemma 8, the existence of six lines in higgledy-piggledy arrangement implies the existence of a resolving set of size $12 q$ at once.

Let us remark that, by Lemma 5 , the smallest size of a set of lines in higgledy-piggledy arrangement in $\mathrm{PG}(4, q)$ is 6 , hence we cannot construct smaller resolving sets in this way.

Finally, our last theorem in this section gives an upper bound in the cases when Theorem 10 cannot be applied.

Theorem 13. If $n>3$, then the graph $\Gamma_{P, \mathcal{H}}(n, q)$ has a resolving set of size $\left(n^{2}+n-8\right) q$. 
Proof. By Lemma 8, it is enough to give a set $\mathcal{L}_{n}$ of $\frac{n^{2}+n-8}{2}$ lines in higgledy-piggledy arrangement.

The construction is given by induction on the dimension. For $n=4$ Theorem 12 guarantees the existence of a required set of points which is contained in the union of 6 lines. Suppose that PG $(k, q)$ admits a set of $\frac{k^{2}+k-8}{2}$ lines that resolves the hyperplanes of the space. Consider PG $(k+1, q)$. Let $\Sigma$ be a hyperplane in $\mathrm{PG}(k+1, q)$. Then, $\Sigma$ is isomorphic to $\mathrm{PG}(k, q)$, hence we can choose a set $\mathcal{L}_{k}$ of $\frac{k^{2}+k-8}{2}$ lines in $\Sigma$ such that no $(k-2)$-dimensional subspace of $\Sigma$ meets each element of $\mathcal{L}_{k}$. Take $k+1$ pairwise skew lines in $\operatorname{PG}(k+1, q)$, say $\ell_{1}, \ell_{2}, \ldots, \ell_{k+1}$, such that each of them intersects $\Sigma$ in exactly one point, and the $k+1$ points $P_{i}=\Sigma \cap \ell_{i}$ generate $\Sigma$. We claim that no $(k-1)$-dimensional subspace of $\mathrm{PG}(k+1, q)$ meets each element of the line-set

$$
\mathcal{L}_{k+1}=\mathcal{L}_{k} \cup\left\{\ell_{1}, \ell_{2}, \ldots, \ell_{k+1}\right\} .
$$

Let $\Pi$ be a $(k-1)$-dimensional subspace of $\mathrm{PG}(k+1, q)$. If $\Pi$ is not contained in $\Sigma$, then $\Pi \cap \Sigma$ is a $(k-2)$-dimensional subspace of $\Sigma$, hence it cannot meet each element of $\mathcal{L}_{k}$, while if $\Pi \subset \Sigma$, then $\Pi$ cannot contain all points $P_{1}, P_{2}, \ldots, P_{k+1}$, hence it cannot meet each of the lines $\ell_{1}, \ell_{2}, \ldots, \ell_{k+1}$.

By definition, the set $\mathcal{L}_{k+1}$ contains

$$
\frac{k^{2}+k-8}{2}+(k+1)=\frac{(k+1)^{2}+(k+1)-8}{2}
$$

lines which proves the theorem.

\section{Resolving sets for small $q$}

We performed a computer search for small resolving sets and semi-resolving sets in $\mathrm{PG}(n, q), n=3,4$ for small values of $q$. All computations have been performed using MAGMA [3].

The results concerning the search for semi-resolving sets are summarized in Table 1, where a dot after a value means that no smaller semi-resolving sets exist, while the superscript indicates the number of equivalence classes up to $\operatorname{PGL}(n+1, q)$.

Classifications and non-existence proofs have been obtained using an exhaustive algorithm. In both PG( $n, 2), n=3,4$, the smallest semi-resolving set is the projective frame. We also proved that no resolving sets of size less than 8 and 10 exist in $\mathrm{PG}(n, 2), n=3,4$, respectively. In $\mathrm{PG}(3,3)$, nine of the semi-resolving sets of size nine are subsets of semi-resolving sets obtained by Theorem 9, while the tenth is an incomplete cap; we also found a resolving set of size 17, consisting of nine points and eight planes. The semi-resolving set of size ten in $\operatorname{PG}(4,3)$ is an incomplete cap. The other examples in Table 1 have been obtained using a randomized greedy algorithm. The 
Table 1: The smallest known sizes of semi-resolving sets in $\mathrm{PG}(n, q), n=3,4$

\begin{tabular}{|c||c|c|c|c|c|}
\hline$q$ & 2 & 3 & 4 & 5 & 7 \\
\hline $\mathrm{PG}(3, q)$ & $4^{1}$. & $9^{10}$. & 13 & 17 & 27 \\
\hline $\mathrm{PG}(4, q)$ & $5^{1}$. & $10^{1}$. & 20 & 28 & 41 \\
\hline
\end{tabular}

semi-resolving set of size 13 in $\mathrm{PG}(3,4)$ is a subset of a semi-resolving set obtained by Theorem 9. All the other examples contained in Table 1 are not subsets of semi-resolving sets obtained by Theorem 9 or by Theorem 12 .

\section{References}

[1] R. F. Bailey and P. J. Cameron: Base size, metric dimension and other invariants of groups and graphs, Bull. London Math. Soc. 43 (2011), 209-242.

[2] D. Bartoli, T. Héger, Gy. Kiss and M. Takáts: On the metric dimension of affine planes, biaffine planes and generalized quadrangles, Australas. J. Comb. 72 (2) (2018), 226-248.

[3] W. Bosma, J. Cannon and C. Playoust: The Magma algebra system. I. The user language, J. Symbolic Comput. 24 (1997), 235-265.

[4] Sz. Fancsali and P. Sziklai: Lines in higgledy-piggledy arrangements, Electron. J. Combin. 21 (2) (2014), Paper 2.56, 15 pp.

[5] T. Héger, B. Patkós and M. Takáts: Search problems in vector spaces, Des. Codes and Cryptography 76 (2015), 207-216.

[6] T. Héger and M. Takáts: Resolving sets and semi-resolving sets in finite projective planes, Electron. J. Combin. 19 (4) (2012), Paper 30, 21 pp.

[7] C. Hernando, M. Mora, I. M. Pelayo, C. Seara and D. R. Wood: Extremal graph theory for metric dimension and diameter, Electron. J. Combin. 17 (2010), R30.

[8] J. W. P. Hirschfeld: Projective Geometries over Finite Fields, $2^{\text {nd }}$ ed., Clarendon Press, Oxford, 1998. 
[9] J. W. P. Hirschfeld and J. A. Thas: General Galois Geometries, Clarendon Press, Oxford, 1991.

[10] C. C. Sims, Determining the conjugacy classes of a permutation group, Computers in Algebra and Number Theory (eds. G. Birkhoff and M. Hall, Jr., American Mathematical Society, Providence, 1971), 191-195.

[11] H. Stichtenoth: Algebraic Function Fields and Codes, 2nd Edition, Graduate Texts in Mathematics, vol. 254, Springer, Berlin, 2009.

Daniele Bartoli, Stefano Marcugini and Fernanda Pambianco

Dipartimento di Matematica e Informatica, Università degli Studi di Perugia

Via Vanvitelli 1, 06123 Perugia, Italy

emails: daniele.bartoli@unipg.it, stefano.marcugini@unipg.it, fernanda.pambianco@unipg.it

György Kiss

Department of Geometry and

MTA-ELTE Geometric and Algebraic Combinatorics Research Group

ELTE Eötvös Loránd University, Budapest,

1117 Budapest, Pázmány Péter sétány 1/C, Hungary, and

FAMNIT, University of Primorska,

6000 Koper, Glagoljaška 8, Slovenia

e-mail: kissgy@cs.elte.hu 